\title{
NEUTRAL HYDROGEN IN GALACTIC CLUSTERS
}

\author{
F. D. DRAKE \\ Harvard College Observatory, Cambridge, Massachusetts, U.S.A.
}

Observations of the neutral hydrogen content of the galactic clusters $h$ and $\chi$ Persei, the Pleiades, the cluster in Coma Berenices, Praesepe, and M 67 have been made with the Harvard College Observatory 60-foot radio telescope. The receiver used was the original receiver made by the Ewen Knight Corporation for the Harvard 24-foot radio telescope. The observations were of three kinds: (1) right-ascension drift curves at $50 \mathrm{kc} / \mathrm{s}$ bandwidth, with the receiver fixed-tuned to the frequency of maximum cluster emission, if known, or to the frequency given by the cluster optical radial velocity; (2) conventional frequency profiles at the position of maximum cluster radiation; (3) "residual" frequency profiles, where there is significant general galactic radiation at the cluster frequency and position. These data provide the spatial distribution of any $\mathrm{H} \mathrm{I}_{\mathrm{I}}$ in the cluster vicinity that is in excess of the general galactic $\mathrm{H} \mathrm{I}_{\mathrm{I}}$, and a frequency profile for this excess hydrogen. Any excess $\mathrm{H}_{\mathrm{I}}$ found is considered associated with the cluster if it agrees in velocity and position with the optical cluster.

A summation of the observational results obtained is given in Table I.

TABLE I

\begin{tabular}{lccccc}
\multicolumn{1}{c}{ Cluster } & Age & $\boldsymbol{M}_{\boldsymbol{H}}$ & $\boldsymbol{M}_{\boldsymbol{H}} / \boldsymbol{M}_{*}$ & $\boldsymbol{M}_{\boldsymbol{H}} / \boldsymbol{M}_{\boldsymbol{p}}$ & $\boldsymbol{D}_{\boldsymbol{H}} / \boldsymbol{D}_{*}$ \\
h, × Persei & $3 \times 10^{\circ} \mathrm{yr}$. & 74000 & 1.05 & 2.1 & 4.7 \\
Pleiades & 150 & 470 & 1.8 & 7.5 & 3.2 \\
Coma Berenices & 700 & 27 & .23 & 2.1 & 1.1 \\
Praesepe & 1000 & 26 & .08 & 0.9 & 1.7 \\
M 67 & 5000 & $<18$ & $<.05$ & - & -
\end{tabular}

$M_{B}$ is the total neutral-hydrogen mass observed in a cluster; $M_{*}$ is the total stellar mass in the cluster; $D_{H}$ is the observed diameter of the cluster hydrogen, after correction for beamwidth; $D_{*}$ is the optical cluster diameter; and $M_{p}$ is the minimum mass of protostars which will be in a cluster according to the ideas of Salpeter and the stellar evolution theorists.

It is seen that appreciable $\mathrm{HI}_{\mathrm{I}}$ exists in all clusters observed except the oldest, M 67. The run of the ratio $M_{H} / M_{*}$ seems to rule out the hypotheses that $M_{B}$ represents gas accreted from the interstellar medium, or gas shed by cluster stars in the course of their evolution. Either of these processes would require an increase in this ratio with cluster age, which is opposite to the observed effect. The hypothesis that the gas is material recently shed by evolving stars and continuously being lost from the clusters seems im- 
probable owing to the large values of $M_{B}$ observed. Any hypothesis assuming continuous loss of mass from the cluster would be permissible only if the mass involved is much smaller than the total cluster mass. A remaining hypothesis fitting both the values of $M_{B}$ and the ratio $M_{B} / M_{*}$ is that the $\mathrm{H}_{\mathrm{I}}$ masses are the residues of the primordial gas masses from which the clusters were formed. The theories of stellar evolution suggest that such gas masses should exist, and that they should have about the observed masses. For these reasons, this hypothesis of the origin of $M_{B}$ is preferred.

It is of interest to compare the values of $M_{B}$ and $M_{p} . M_{p}$ is found by assuming, following the theories of stellar evolution, that all those cluster gas masses that are still in the protostellar state will produce stars whose visual absolute magnitudes are eight magnitudes or more fainter than the upper main sequence turnoff. The number of such objects is found by fitting the Salpeter "formation" function to the observed optical-cluster luminosity functions. If $M_{\boldsymbol{H}}$ does consist of the residue of the primordial cluster mass, fragmented into protostars that will eventually form stars according to the Salpeter function, then we would expect $M_{H} / M_{p}$ to be of the order of one, but greater than one. $M_{H} / M_{p}$ should be greater than one in this case because apparently a protostar must shed material while condensing to the stellar state. We are assuming here that essentially all the mass of a protostar is in the form of neutral atomic hydrogen of low optical depth. We see that the values of $M_{H} / M_{\mathrm{p}}$ do fall near the expected values. This supports the idea that we are dealing with protostars, and also supports the idea lying behind the Salpeter function: that the relative numbers of stars of different masses formed from a given mass of gas are the same everywhere in the Galaxy.

Accurate frequency profiles have been obtained for the hydrogen in the Pleiades and Praesepe. The profiles are nearly identical in form, having extended wings which give the line profile the characteristic appearance of a damping profile. The r.m.s. frequency displacement is about $32 \mathrm{kc} / \mathrm{s}$. It may be shown that the line-broadening cannot be the result of collisional damping due either to inter-atom collisions or to scattering of Lyman alpha quanta. The line-broadening may be caused by Zeeman effect only if a rather implausible model for the gas masses is assumed. Conventional Doppler broadening appears at present to offer the best explanation of the line profile.

The acceptance of Doppler effect as the source of line-broadening, however, offers a difficulty in that the r. m. s. velocity implied, $6.8 \mathrm{~km} / \mathrm{second}$, greatly exceeds the cluster escape velocity of about $1 \mathrm{~km} / \mathrm{second}$. Rapid escape of all cluster gas masses should occur if no constraining force other than gravity exists in the cluster. Such an additional constraining force may be provided by an intrinsic cluster magnetic field, $H_{c}$. In such a case, we will find that the cluster gas masses and magnetic field form a magnetohydrodynamic system in which there is an approach to equipartition of energy between gas masses and field. This system, in turn, will be constrained by the magnetic pressure of the general galactic magnetic field, $H_{g}$. It may be shown that 
these hypotheses lead to the following relations between the field strengths required and the observational $21-\mathrm{cm}$ parameters:

$$
H_{g} \approx 1.3 H_{c} \text {, }
$$

where

$$
H_{c} \approx 3 M_{B^{1 / 2}} v_{1} R^{-3 / 2} .
$$

$v_{1}$ is the r.m.s. longitudinal gas velocity component, and $R$ is the radius of the cluster gas system. Inserting the observed values of $M_{H}, R$, and $v_{1}$, we obtain,

$$
\begin{aligned}
\text { near the Pleiades, } H_{g} \approx 2.6 \times 10^{-5} \text { gauss, } \\
\text { near Praesepe, } H_{o} \approx 1.7 \times 10^{-5} \text { gauss. }
\end{aligned}
$$

These results agree very well with the previous estimates of the general galactic field strength based on quite different considerations; they thus support the interpretation discussed here, and provide a new measure of the general galactic magnetic field strength.

Additional support for this interpretation is given by the run of the ratio $D_{B} / D_{*}$. Let us assume that gas is being removed from the cluster magnetohydrodynamic system owing to the formation of new stars or some other process. Whenever this occurs, the pressure in the magnetohydrodynamic system decreases, and no longer balances the galactic pressure. The galactic pressure then compresses the system, increasing the pressure caused by both cluster magnetic field and gas masses, until equilibrium is again attained. In the light of this, we should expect to find $D_{H}$ decreasing with time, or in general, a decrease in the ratio $D_{H} / D_{*}$ with increasing cluster age. Such a qualitative effect is shown in the results of Table $I$, which, along with the quantitative agreement with these ideas shown by the Pleiades and Praesepe, lends further support to the interpretation presented here.

\section{Discussion}

Schmidt: I wonder whether Dr. Erickson would care to tell us about Dr. Helfer's recent observations on the Pleiades?

Erickson: Helfer has made a general survey of the Pleiades region. He finds the whole area of sky near the cluster to be exceedingly complex, possessing at least three or perhaps four clouds, none of which coincides either in position or in velocity with the optical cluster. In fact, the cluster lies in a trough between two of the clouds. Most of the comparison points used by Drake also lie in troughs between clouds. Helfer finds no distinct neutral hydrogen gas cloud that he can definitely associate with the optical cluster.

Drake: Helfer's conventional-frequency profile observations of the Pleiades are objectionable because they require a subjective decision as to which portion of the profile belongs to the cluster and which to the general galactic radiation. Heeschen and I had found this in our 1955 study of the Pleiades, and this led to the use of drift curves in the present study. Drift curves 
separate cluster and general radiation components with complete objectivity, and, as seen on the original radiometer tracings, show a marked $\mathrm{H}$ I concentration at the cluster position. With regard to the suggestion that there is a ringlike trough in the $\mathrm{HI}$ density surrounding the Pleiades, this would lead to a residual profile which would be only a lower-intensity duplicate of the general profile in the region. Such is clearly not the case; with every comparison position the residual profile has a sharp peak at about $-1 \mathrm{~km} / \mathrm{second}$ and no component at $+6 \mathrm{~km} / \mathrm{second}$, where the general profile has a peak. This indicates no trough, but rather the existence of cluster hydrogen at about $-1 \mathrm{~km} / \mathrm{second}$. The velocity of the final residual profile is $-1.2 \mathrm{~km} / \mathrm{sec}$ ond, in exact agreement with the optical cluster velocity. It seems quite unlikely that the above is purely a chance coincidence.

Oort: Doesn't the large hydrogen mass that you find in the Pleiades conflict with the observed internal motions of the stars?

Drake: These results seem not to conflict with the dynamically determined cluster masses. The large $\mathrm{H}_{\mathrm{I}}$ diameters mean that only small, permissible portions of the $\mathrm{HI}_{\mathrm{I}}$ masses lie within the optical clusters where they may affect the stellar motions. Furthermore, it appears that the gas masses have no significant effect on the stellar dynamics. The strong dependence of the relaxation time on velocity results in relaxation times of the order of $10^{9}$ years for the transfer of energy from the gas masses to the stars. Thus the stars "feel" the presence of the gas masses almost not at all.

Oort: The optical diameter of 2 degrees which you quote for the Pleiades probably refers to the brightest stars. Certainly, the stars down to the 15th magnitude show a considerably larger diameter.

Drake: The optical cluster diameters have been taken from the usual sources. They may well be in error, but no change in the conclusions is necessary as long as the errors are systematic and not too large. 